\title{
Energy-Filtered Transmission Electron Microscope Tomography of Silicon Nanoparticles in Silicon Dioxide Deposited with High Density Plasma Chemical Vapor Deposition
}

\author{
T. Brintlinger ${ }^{1}$, M. E. Twigg ${ }^{2}$, and H. L. Hughes ${ }^{1}$ \\ 1. Material Science and Technology Division, U. S. Naval Research Laboratory, Washington, DC \\ 2. Electronics Science and Technology Division, U. S. Naval Research Laboratory, Washington, DC
}

High Density Plasma Chemical Vapor Deposition (HDPCVD), a variant of Plasma-Enhanced Chemical Vapor Deposition (PECVD), has demonstrated the capability of growing $\mathrm{SiO}_{2}$ layers with novel electronic properties. In particular, the structure of Si nanoparticles (NPs), in Si-rich HDPCVD $\mathrm{SiO}_{2}$ layers has been correlated with the performance of metal oxide semiconductor (MOS) memory devices [1]. There remains, however, a need to understand the underlying thermodynamics and kinetics of the HDPCVD process, especially as it relates to evolution of the size, shape, and density of Si NPs in $\mathrm{SiO}_{2}$ [2]. Such parameters as the deposition rate and the relative flow rate of reagents (e.g. $\mathrm{SiH}_{4}$ and $\mathrm{N}_{2} \mathrm{O}$ ) [3], and their influence on Si NP configuration have been shown to play a vital role. In order to properly assess the influence of deposition parameters on the evolution of Si NPs, a detailed characterization of Si NP size and shape is essential. To that that end, Energy-Filtered Transmission Electron Microscopy (EFTEM) has been used to map the intensity of the $17 \mathrm{eV}$ Si plasmon, in order to reveal the presence of $\mathrm{Si} N \mathrm{NS}$ in the $\mathrm{SiO}_{2}$ layer [4]. Furthermore, a more complete characterization of the complex structure of the Si NPs can be achieved by EFTEM tomography, which provides a 3-dimensional (3D) view of the NP-rich regions of the $\mathrm{SiO}_{2}$ layer [5-7].

Here, EFTEM tomography has been used to provide a 3D map of the Si NPs in the $\mathrm{SiO}_{2}$ layer within silicon on insulator (SOI) material. For this study, we use a JEM2200FS together with an "in-lens" filter and Ultrascan camera. Following extraction of cross-section containing Si NPs in $\mathrm{SiO}_{2}$, EFTEM images are acquired in $4^{\circ}$ increments with an $8 \mathrm{eV}$ slit centered on the $17 \mathrm{eV} \mathrm{Si}$ plasmon, and then assembled using ETomo software package with tomograms generated using back-projection. Following tomogram generation, silica NPs are filtered and segmented using Avizo software (Fire 8.0). Resulting equivalent diameter distribution shows a fairly broad, flat distribution in Si NP equivalent diameter, with a mean of $\sim 25 \mathrm{~nm}$ [8]. A detailed examination of the Si NP morphology reveals that many of the NPs are extended parallel to the $\mathrm{Si} / \mathrm{SiO}_{2}$ interface. Such an extension suggests phase separation beyond that occurring during deposition, and which may be attributed to spinoidal decomposition [2]. The incidence of this mode of post-growth phase separation suggests the ability to alter Si NP morphology though the control of post-growth annealing.

\section{References}

[1] G Chakraborty, et al., Journal of Applied Physics 109 (2011), p. 064504.

[2] T Müller, KH Heinig \& W Möller, Materials Science and Engineering: B 101 (2003), p. 49-54.

[3] F Ehrhardt, et al., physica status solidi (c) 9 (2012), p. 1878-1883.

[4] C Spinella, et al., Appl Phys Lett 87 (2005), p. 044102.

[5] D Friedrich, et al., Appl Phys Lett 103 (2013), p. 131911

[6] LF Kourkoutis, et al., Nanoscale 5 (2013), p. 7499-7504.

[7] A Yurtsever, M Weyland \& DA Muller, Appl Phys Lett 89 (2006), p. 151920.

[8] Work at the Naval Research Laboratory was funded through the Office of Naval Research. 


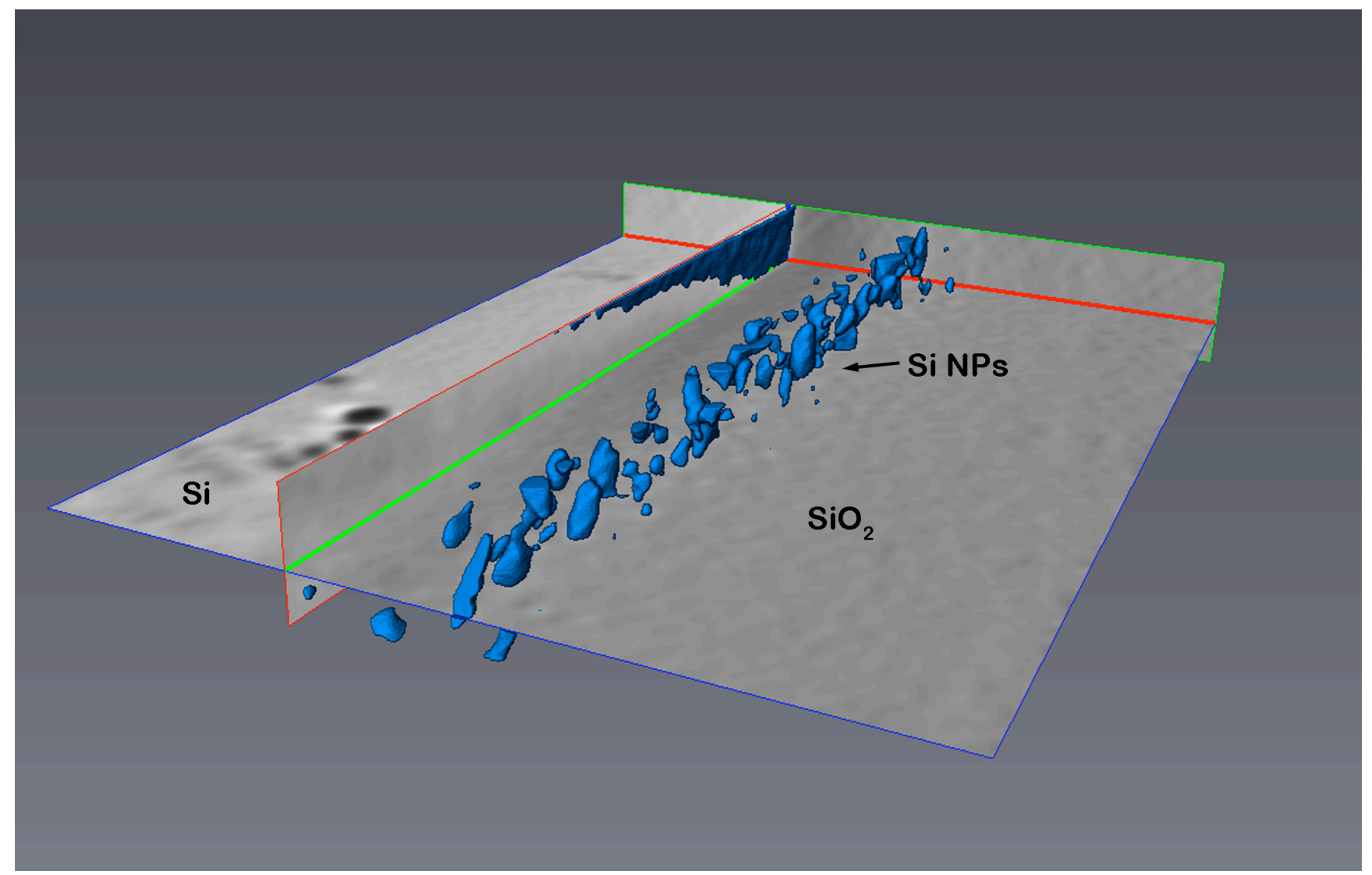

Figure 1. Segmented tomogram showing $\mathrm{Si}$ NPs in $\mathrm{SiO}_{2}$. Linear slice parallel to $\mathrm{Si}$ NPs (with red outline) represents original $\mathrm{SiO}_{2}$ surface. For scale, NPs are $\sim 20 \mathrm{~nm}$ in diameter.

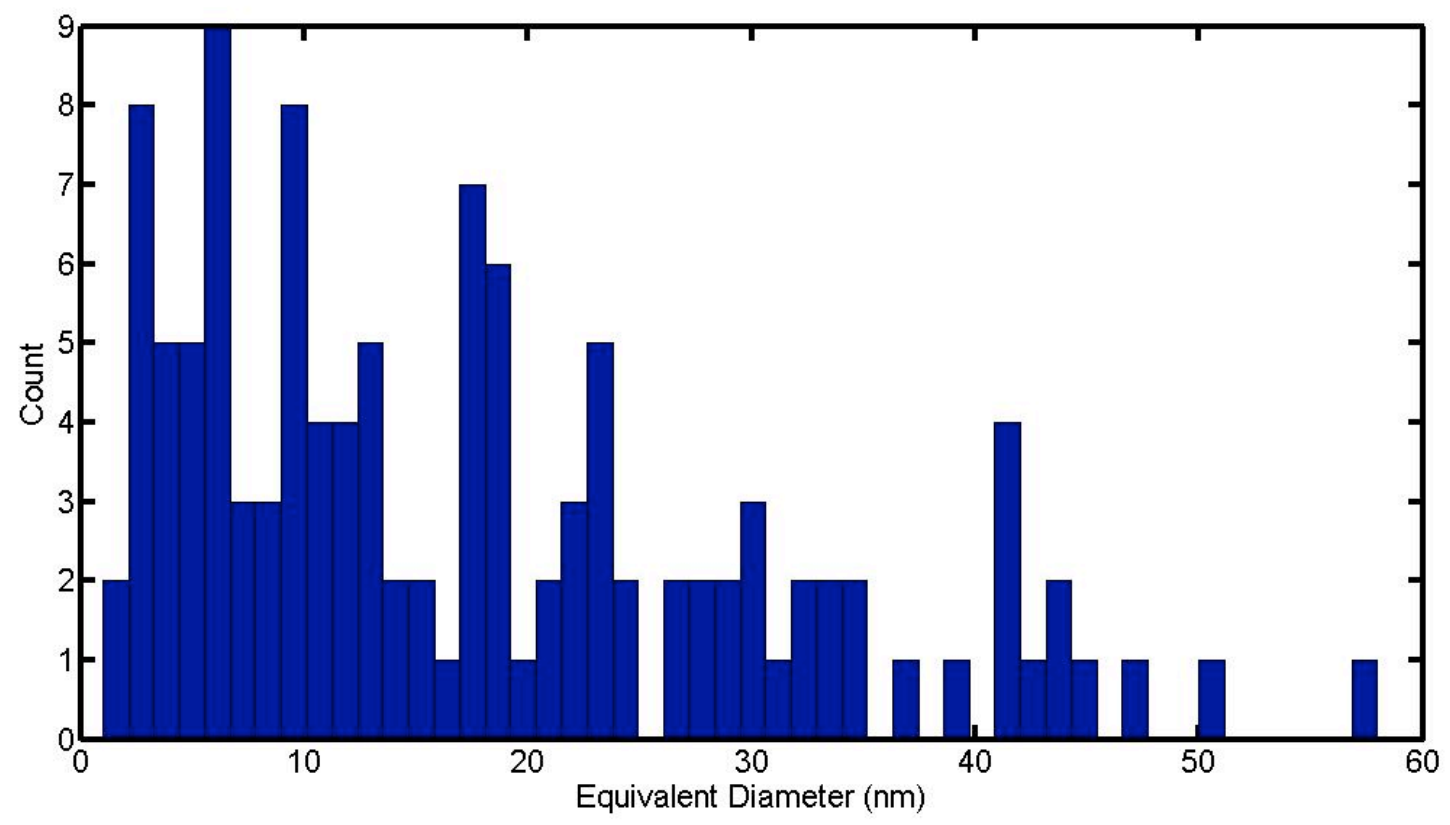

Figure 2. Histogram of equivalent diameter of segmented Si NPs in Fig. 1. 